\title{
DYNAMIC BEHAVIOUR OF THREE-LAYERED ANNULAR PLATES WITH VISCOELASTIC CORE UNDER LATERAL LOADS
}

\author{
Dorota PaWlus \\ University of Bielsko-Biala, Faculty of Mechanical Engineering and Computer Science, Bielsko-Biata, Poland \\ e-mail:dpawlus@ad.ath.bielsko.pl
}

\begin{abstract}
This paper presents the dynamic behaviour of three-layered, annular plates with a linear viscoelastic core. The annular plate is loaded in plane of facings with variable in time forces acting on the inner or outer plate edges. The analysed plate structure is symmetric and composed of three-layers: thin facings and a thicker, soft core. The core material is considered as linear with viscoelastic properties. Analytical and numerical solution will be presented including the wavy forms of dynamic loss of plate stability. In the analytical and numerical solution, two approximation methods: orthogonalization and finite difference have been applied to obtain the system of differential equations for the analysed problem of dynamic deflections of the examined plate with the viscoelastic core. Additionally, the suitable plate model using the finite element method has been built. The numerical calculations carried out in ABAQUS system show the results which are compatible with those obtained for the analytical and numerical solution. Presented in figures time histories of plate deflections and velocity of deflections as well as buckling forms of the plate model show the dynamic response of the examined plate on time-dependent loads and core material properties.
\end{abstract}

Keywords: sandwich plate, viscoelastic core, dynamic stability

\section{Introduction}

The examinations of three-layered plates have been undertaken in numerous works. Rarely, they concern annular plates under lateral loads variable in time and plate structures where damping properties of the layer material is taken into account. A wide range of possible applications of annular layered plates in aerospace industry, mechanical and nuclear engineering presented in works by Dumir and Shingal (1985), Paydar (1990), Chen et al. (2006), Wang and Chen (2004) causes the evaluation of dynamic behaviour of such complex composite plates still important.

The following, exemplary papers by Wang and Chen (2002, 2004), Chen et al. (2006) belong to the group of works, where the plate dynamic problems like vibrations or dynamic instability have been analysed. The examinations concern the plates composed of three layers: the thin outer constraining layer, the thin middle viscoelastic damping layer and the bottom outer host layer of the plate. The evaluation of the influence of the viscoelastic core thickness on the improvement of damping plate properties was presented in work by Wang and Chen (2002). In many works, the problem of the loss of plate stability wss often limited to only the axisymmetric case. Whereas, full evaluation also requires analysis of circumferentially waved plate buckling forms. In the work by Krizhevsky and Stavsky (1996), the asymmetric instability problem of a three-layered plate with a relatively thin core was shown. The results of critical loads were presented.

The evaluation of dynamic behaviour of a three-layered annular plate with a viscoelastic core loaded in facings plane is presented in this work. The type of the three-layered structure is characteristic, transversally symmetrical and composed of thin facings and a suitable thicker, soft core. The asymmetric buckling modes of examined, dynamic loaded plates are also observed. 
Including in the description of physical relations the rheological properties of the plate core made of polyurethane foam correctly approximates the critical real plate behaviour. It could be supposed that the noticed in the work by Romanów (1995) sensitivity of foam materials on the long duration of load could be important particularly in supercritical plate work under a load constant in time or slowly increasing.

Such observations together with dynamic stability analyses of three-layered annular plates with viscoelastic cores are the main goal of this work. The presented way of problem solution and detailed results refer to analyses presented in works by Pawlus (2010, 2011a,b,c).

\section{Problem formulation}

The three-layered annular plate with the soft foam core and with the symmetric cross-section structure is the subject of consideration. The scheme of the plate is presented in Fig. 1. The facings are elastic. The core material is treated as elastic or viscoelastic. The plate is loaded on the inner or/and outer perimeter of facings with uniformly distributed stress linearly increasing in time, according to Equation (2.1) or constant in time (see, Eq. (6.1))

$$
p=s t
$$

where $p$ is compressive stress, $s$ - rate of growth of the plate loading, $t$ - time.

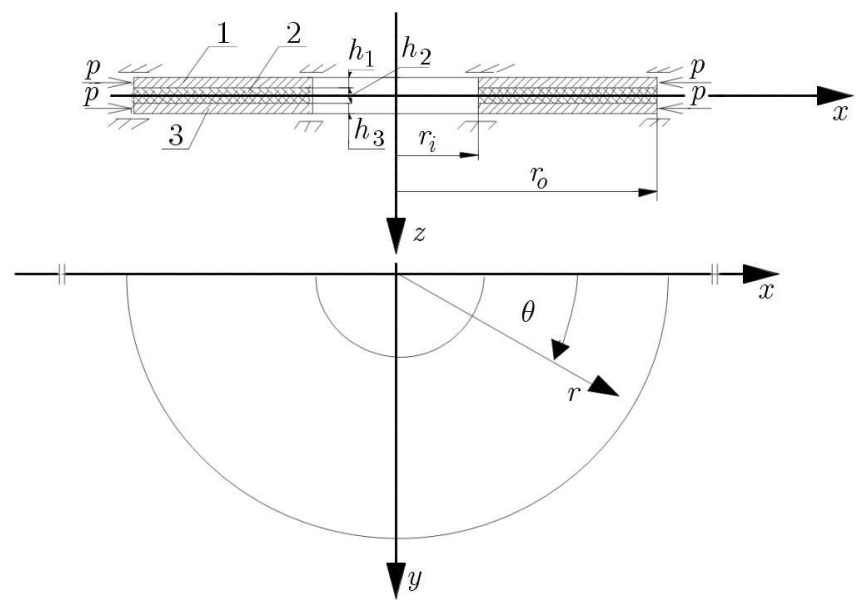

Fig. 1. A scheme of the three-layered annular plate composed of facings - layers 1, 3 and core - layer 2

The plate with bilaterally slidably clamped edges is undertaken in the presented considerations. The form of plate predeflection corresponds with the plate buckling form. The circumferentially waved forms of the loss of plate dynamic stability are analysed, too. The classical theory of sandwich plates with the broken line hypothesis, presented by Volmir (1967), and the decomposition of basic stresses to normal and shearing components, carried by facings and the core, respectively, have been accepted in the mathematical formulation of the problem. In numerical solution, the approximate finite difference method and finite element method have been used.

As the criterion of the loss of plate stability, the criterion presented by Volmir (1972) has been adopted. According to this criterion, the loss of plate stability occurs at the moment of time when the speed of the plate point of the maximum deflection reaches the first maximum value. The essence of the accepted criterion of the loss of plate stability connected with the evaluation of values of dynamic critical loads in the region of significant increase in plate deflections corresponds to the Budiansky-Roth criterion. This criterion is used in the buckling analysis of laminated shells, presented for example by Tanov and Tabiei (1998). 


\section{Basic equations}

Solution to the considered problem is based on the relations typical for the edge-initial problem: dynamic equilibrium equations, geometrical and physical relations and initial and boundary conditions. Dynamic equilibrium equations have been formulated for each layer of the plate. Core deformation is described by the angles $\beta$ and $\alpha$ in the radial and circumferential directions, respectively

$$
\begin{aligned}
& \beta=\frac{u_{1}-u_{3}}{h_{2}}-\frac{1}{2} \frac{h_{1}+h_{3}}{h_{2}} w_{d, r}+w_{o, r} \\
& \alpha=\frac{v_{1}-v_{3}}{h_{2}}-\frac{1}{2 r} \frac{h_{1}+h_{3}}{h_{2}} w_{d, \theta}+\frac{1}{r} w_{o, \theta}
\end{aligned}
$$

where $w_{d}$ is additional plate deflection, $w_{o}$ - preliminary plate deflection, $u_{1(3)}, v_{1(3)}$ - displacements of the points of the middle plane of facings (layers 1,3) in the radial and circumferential directions, respectively.

Linear physical relations of Hooke's law for facings and linear viscoelastic relations of the standard model (see Fig. 2) for the plate core are applied. The physical relations of the viscoelastic core material subjected to shearing stresses are presented by the equations

$$
\tau_{r z_{2}}=\widetilde{G}_{2} \gamma_{r z_{2}} \quad \tau_{\theta z_{2}}=\widetilde{G}_{2} \gamma_{\theta z_{2}}
$$

where $\gamma_{r z_{2}}, \gamma_{\theta z_{2}}$ are shearing strains of the plate core in the radial and circumferential direction, respectively, expressed by

$$
\gamma_{r z_{2}}=u_{2, z}^{(z)}+w_{d, r} \quad \gamma_{\theta z_{2}}=v_{2, z}^{(z)}+\frac{1}{r} w_{d, \theta}
$$

where: $u_{2}^{(z)}=u_{2}-z \beta+z w_{o, r}, v_{2}^{(z)}=v_{2}-z \alpha+z w_{o, \theta} / r$ are the radial and circumferential displacements of the points in the distance $z$ between the point and the middle surface of the plate core; $u_{2}, v_{2}$-displacements of the points of the middle plane of the core layer in the radial and circumferential direction, respectively; $\widetilde{G}_{2}$ - modulus expressed by the formula corresponding to the form of the constitutive equation of the standard model

$$
\widetilde{G}_{2}=\frac{C+D \frac{\partial}{\partial t}}{E+F \frac{\partial}{\partial t}}
$$

where $C, D, E, F$ are the quantities formulated by the elastic $G_{2}, G_{2}^{\prime}$ and viscosity $\eta^{\prime}$ constants of the core material, presented by Skrzypek (1986)

$$
C=\frac{G_{2} G_{2}^{\prime}}{G_{2}+G_{2}^{\prime}} \quad D=\frac{\eta^{\prime} G_{2}}{G_{2}+G_{2}^{\prime}} \quad E=1 \quad F=\frac{\eta^{\prime}}{G_{2}+G_{2}^{\prime}}
$$

and $\partial / \partial t$ - differential operator.

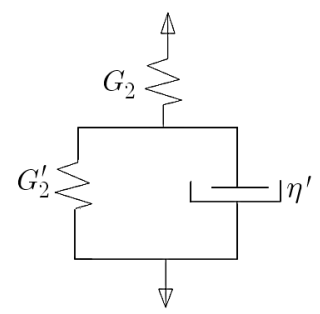

Fig. 2. The standard model of a viscoelastic layer material 
Using the equations of the nonlinear Kármán's plate, the sectional forces and moments in the facings are established. Then, the resultant transverse radial and circumferential forces and the resultant membrane forces expressed by the introduced stress function are formulated.

The initial conditions, loading and boundary conditions as well as relations connected with the slidably clamped both inner and outer plate edges are established as follows

$$
\begin{array}{lccc}
\left.w\right|_{t=0}=w_{o} & \left.w_{, t}\right|_{t=0}=0 & \left.w_{d}\right|_{t=0}=0 & \left.w_{d, t}\right|_{t=0}=0 \\
\left.\sigma_{r}\right|_{r=r_{i}\left(r_{o}\right)}=-p(t) d_{1(2)} & \left.\sigma_{r, t}\right|_{r=r_{i}\left(r_{o}\right)}=-(p(t))_{, t} d_{1(2)} & \left.\tau_{r \theta}\right|_{r=r_{i}\left(r_{o}\right)}=0 \\
\left.w\right|_{r=r_{i}\left(r_{o}\right)}=0 & \left.w_{, r}\right|_{r=r_{i}\left(r_{o}\right)}=0 & \delta=\left.\gamma\right|_{r=r_{i}\left(r_{o}\right)}=0 & \left.\delta_{, r}\right|_{r=r_{i}\left(r_{o}\right)}=0
\end{array}
$$

where $w$ is the total deflection, $w_{d}$ - additional deflection, $w_{o}$ - preliminary deflection, $\sigma_{r}$ - radial stress, $\tau_{r \theta}$ - shear stress, $d_{1}, d_{2}$ - quantities equal to 0 or 1 , determining the loading of the inner or/and outer plate perimeter, $\delta, \gamma$-differences of radial and circumferential displacements of the points in middle surfaces of facings, respectively.

Finally, after some calculations the basic differential equation expressed the deflections of the analysed sandwich plate with the viscoelastic core are determined

$$
\begin{aligned}
& N_{1} w_{d, r r r r}+\frac{2 N_{1}}{r} w_{d, r r r}-\frac{N_{1}}{r^{2}} w_{d, r r}+\frac{N_{1}}{r^{3}} w_{d, r}+\frac{N_{1}}{r^{4}} w_{d, \theta \theta \theta \theta}+\frac{2\left(N_{1}+N_{2}\right)}{r^{4}} w_{d, \theta \theta} \\
& +\frac{2 N_{2}}{r^{2}} w_{d, r r \theta \theta}-\frac{2 N_{2}}{r^{3}} w_{d, r \theta \theta}-\widetilde{G}_{2} \frac{H^{\prime}}{h_{2} r}\left(\gamma_{, \theta}+\delta+r \delta_{, r}+H^{\prime} \frac{1}{r} w_{d, \theta \theta}+H^{\prime} w_{d, r}+H^{\prime} r w_{d, r r}\right) \\
& =\frac{2 h^{\prime}}{r}\left(\frac{2}{r^{2}} \Phi_{, \theta} w_{, r \theta}-\frac{2}{r} \Phi_{, r \theta} w_{, r \theta}+\frac{2}{r^{2}} w_{, \theta} \Phi_{, r \theta}-\frac{2}{r^{3}} w_{, \theta} \Phi_{, \theta}+w_{, r} \Phi_{, r r}+\Phi_{, r} w_{, r r}\right. \\
& \left.+\frac{1}{r} \Phi_{, \theta \theta} w_{, r r}+\frac{1}{r} \Phi_{, r r} w_{, \theta \theta}\right)-M w_{d, t t}
\end{aligned}
$$

where $N_{1}, N_{2}$ are expressed by the material and geometrical parameters of plate layers; $H^{\prime}=h^{\prime}+h_{2} ; h^{\prime}\left(h_{1}=h_{3}=h^{\prime}\right)-$ facing thickness, $h_{2}$ - core thickness.

\section{Problem solution}

In the solution, the following dimensionless quantities and the expressions have been assumed

$$
\begin{array}{lccc}
\zeta=\frac{w}{h} & \zeta_{1}=\frac{w_{d}}{h} & \zeta_{o}=\frac{w_{o}}{h} & F=\frac{\Phi}{E h^{2}} \\
\rho=\frac{r}{r_{o}} & \bar{\delta}=\frac{\delta}{h} \quad \bar{\gamma}=\frac{\gamma}{h} & t^{*}=t K_{7} & K_{7}=\frac{s}{p_{c r}} \\
\zeta_{1}(\rho, \theta, t)=X_{1}(\rho, t) \cos (m \theta) & \zeta_{o}(\rho, \theta)=X_{a}(\rho)+X_{b}(\rho) \cos (m \theta) \\
\zeta=\zeta_{1}+\zeta_{o} \quad \quad F(\rho, \theta, t)=F_{a}(\rho, t)+F_{b}(\rho, t) \cos (m \theta)+F_{c}(\rho, t) \cos (2 m \theta) \\
\bar{\delta}(\rho, \theta, t)=\bar{\delta}(\rho, t) \cos (m \theta) & \bar{\gamma}(\rho, \theta, t)=\bar{\gamma}(\rho, t) \sin (m \theta)
\end{array}
$$

where $m$ is the number of circumferential waves corresponding to the form of plate buckling, $h=h_{1}+h_{2}+h_{3}$ is the total thickness of plate, $p_{c r}$ - critical static stress.

Using the orthogonal method to elimination of the angular variable $\theta$, Eq. (3.7) has been replaced by the approximate one obtained from the following condition

$$
\int_{0}^{2 \pi} \psi \cos (m \theta) d \theta=0
$$

where $\psi$ is the difference of the left and right side of transformed Equation (3.7). 
Using the physical relations, relations of sectional forces and moments in the facings with stresses and equations of resultant membrane forces expressed by the introduced stress function after elimination of the quantities described by sums of the radial $\left(u_{1}+u_{3}\right)$ and circumferential $\left(v_{1}+v_{3}\right)$ facing displacements, the following equation has been obtained

$$
\begin{aligned}
\Phi_{, r r r r} & +\frac{2}{r} \Phi_{, r r r}-\frac{1}{r^{2}} \Phi_{, r r}+\frac{1}{r^{3}} \Phi_{, r}+\frac{1}{r^{4}} \Phi_{, \theta \theta \theta \theta}+\frac{4}{r^{4}} \Phi_{, \theta \theta}-\frac{2}{r^{3}} \Phi_{, r \theta \theta}+\frac{2}{r^{2}} \Phi_{, r r \theta \theta} \\
= & E\left[\frac{1}{r} w_{o, r r}\left(w_{o, r}+\frac{1}{r} w_{o, \theta \theta}\right)-\frac{1}{r^{2}}\left(\frac{1}{r} w_{o, \theta}-w_{o, r \theta}\right)^{2}-\frac{1}{r} w_{, r r}\left(w_{, r}+\frac{1}{r} w_{, \theta \theta}\right)\right. \\
+ & \left.\frac{1}{r^{2}}\left(\frac{1}{r} w_{, \theta}-w_{, r \theta}\right)^{2}\right]
\end{aligned}
$$

The quantities $\delta$ and $\gamma$, unknown in Equation (3.7), have been obtained determining the differences of the radial and circumferential displacements $u_{1}, u_{3}$ and $v_{1}, v_{3}$ of the points of the middle surface of the plate facings. The equilibrium equations for forces acting on the non-deformed outer plate layers in the $u$ and $v$ direction are used.

After insertion of Equations (4.1) describing the functions $w, w_{o}, \Phi$ and after comparison of the expressions by the same trigonometric functions, Equation (4.3) has been presented in form of three equations.

In the solution, the finite difference method has been used for the approximation of the derivatives with respect to $\rho$ by central differences in the discrete points. The form of the system of differential equations expressing the deflections of three-layered annular plate with the viscoelastic core is as follows

$$
\begin{aligned}
& \mathbf{P U}+\mathbf{Q}+\mathbf{P}_{L} \dot{\mathbf{U}}+\mathbf{Q}_{L}-W_{1} \ddot{\mathbf{U}}=W_{2} \ddot{\mathbf{U}} \\
& \mathbf{M}_{Y} \mathbf{Y}=\mathbf{Q}_{Y} \quad \mathbf{M}_{Y} \dot{\mathbf{Y}}=\dot{\mathbf{Q}}_{Y} \\
& \mathbf{M}_{V} \mathbf{V}=\mathbf{Q}_{V} \quad \mathbf{M}_{V} \dot{\mathbf{V}}=\dot{\mathbf{Q}}_{V} \\
& \mathbf{M}_{Z} \mathbf{Z}=\mathbf{Q}_{Z} \quad \mathbf{M}_{Z} \dot{\mathbf{Z}}=\dot{\mathbf{Q}}_{Z} \\
& \mathbf{M}_{D L} \dot{\mathbf{D}}=\mathbf{M}_{D} \mathbf{D}+\mathbf{M}_{U} \mathbf{U}+\mathbf{M}_{U L} \dot{\mathbf{U}}+\mathbf{M}_{G} \mathbf{G}+\mathbf{M}_{G L} \dot{\mathbf{G}} \\
& \mathbf{M}_{G G L} \dot{\mathbf{G}}=\mathbf{M}_{G G} \mathbf{G}+\mathbf{M}_{G U} \mathbf{U}+\mathbf{M}_{G U L} \dot{\mathbf{U}}+\mathbf{M}_{G D} \mathbf{D}+\mathbf{M}_{G D L} \dot{\mathbf{D}}
\end{aligned}
$$

where

$$
W_{1}=K_{7} \frac{h^{\prime}}{h} r_{o} h_{2} M E_{L} \quad W_{2}=K_{7}^{3} \frac{h^{\prime}}{h} r_{o} h_{2} M F_{L}
$$

and $M$ is the expression: $M=2 h^{\prime} \mu+h_{2} \mu_{2} ; \mu, \mu_{2}$ - facing and core mass density, respectively; $r_{o}$ - outer radius; $E_{L}, F_{L}$ - quantities determined by elastic and viscosity constants of the core standard material; $\mathbf{U}, \mathbf{Y}, \mathbf{V}, \mathbf{Z}, \dot{\mathbf{U}}, \ddot{\mathbf{U}}, \ddot{\mathbf{U}}, \dot{\mathbf{Y}}, \dot{\mathbf{V}}, \dot{\mathbf{Z}}$ - vectors of plate additional deflections and components $F_{a}, F_{b}, F_{c}$ of the stress function $F_{a^{\prime} \rho}=y, F_{b}=v, F_{c}=z$ and their derivatives with respect to time $t$, respectively; $\mathbf{P}, \mathbf{P}_{L}$ - matrices with elements composed of geometric and material plate parameters, the quantity $b(b$ - length of the interval in the finite difference method), dimensionless radius $\rho$ and the number $m$ of buckling waves and its derivative with respect to time $t$, respectively; $\mathbf{Q}, \mathbf{Q}_{L}$ - vectors of expressions composed of the initial and additional deflections, geometric and material parameters, components of the stress function, radius $\rho$, quantity $b$, coefficients $\delta, \gamma$ and the number $m$ and their derivatives with respect to time $t$, respectively; $\mathbf{M}_{Y}$ - matrix of elements composed of the radius $\rho$ and quantity $b$, respectively; $\mathbf{M}_{V}, \mathbf{M}_{Z}$ - matrices of elements composed of the radius $\rho$, quantity $b$ and number $m$; $\mathbf{Q}_{Y}, \mathbf{Q}_{V}, \mathbf{Q}_{Z}, \dot{\mathbf{Q}}_{Y}, \dot{\mathbf{Q}}_{V}, \dot{\mathbf{Q}}_{Z}$ - vectors of expressions composed of the initial and additional deflections, radius $\rho$, quantity $b$ and the number $m$ and their derivatives with respect to time $t$, respectively; $\mathbf{M}_{D}, \mathbf{M}_{G}, \mathbf{M}_{G G}, \mathbf{M}_{G D}, \mathbf{M}_{D L}, \mathbf{M}_{G L}, \mathbf{M}_{G G L}, \dot{\mathbf{M}}_{G D L}$ - matrices of elements composed of geometric and material parameters, quantity $b$ and the number $m$ and their derivatives 
with respect to time $t$, respectively; $\mathbf{M}_{U}, \mathbf{M}_{U L}$ - matrices of elements composed of material parameters and quantity $b$ and their derivatives with respect to time $t$, respectively; $\mathbf{M}_{G U}, \mathbf{M}_{G U L}-$ matrices of elements composed of geometric parameters, material parameters and the number $m$ and their derivatives with respect to time $t$, respectively; $\mathbf{D}, \mathbf{G}, \dot{\mathbf{D}}, \dot{\mathbf{G}}$ - vectors of expressions composed of coefficients $\delta$ and $\gamma$ and their derivatives with respect to time $t$, respectively.

The system of Equations (4.4) has been solved using Runge-Kutta's integration method for the initial state of plate.

Eliminating from the system of Equations (4.4) Equations (4.4) $3,5,7$ and the expressions connected with the differential operator $\partial / \partial t$ applied in physical relations of the viscoelastic material of the plate core, a system of equations for plate with elastic core has been obtained (Pawlus, 2010, 2011b,c). The critical static stress $p_{c r}$ has been calculated solving the eigenproblem for the problem of the disk state neglecting the inertial components and nonlinear expressions (Pawlus, 2010, 2011b,c).

\section{Finite element plate models}

In the analysis carried out with use the finite element method, two models have been built:

- basic model in form of the complete model of the annular plate, circularly symmetrical (Fig. 3a),

- simplified model built of axisymmetrical elements (Fig. 3b).

The facings are built of shell elements but the core mesh is built of solid elements. The grids of facings elements are tied with the grid of core elements using the surface contact interaction. The calculations were carried out at the Academic Computer Center CYFRONET-CRACOW (KBN/SGI_ORIGIN_2000/PŁódzka/030/1999) using the ABAQUS system.

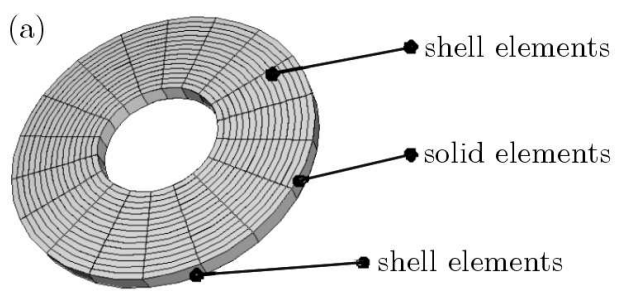

(b)

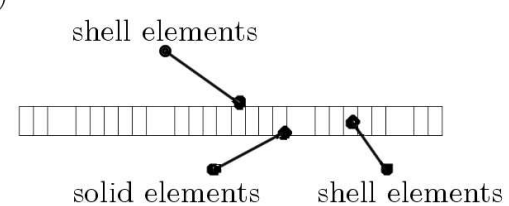

Fig. 3. (a) Basic model, (b) simplified model

The viscoelastic properties of the core material have been described by a single term of the Prony series for the shear relaxation modulus (Hibbitt et al., 2000)

$$
G_{R}(t)=G_{o}\left(1-q_{1}^{p}\left(1-\mathrm{e}^{-t / \tau_{1}^{G}}\right)\right)
$$

where $q_{1}^{p}, \tau_{1}^{G}$ are material constants, $G_{o}$ - instantaneous shear $G_{2}$.

The values of material constants $q_{1}^{p}, \tau_{1}^{G}$ for the standard model of the plate core have been calculated from the following equations

$$
q_{1}^{p}=\frac{G_{2}}{G_{2}+G_{2}^{\prime}} \quad \tau_{1}^{G}=\frac{\eta^{\prime}}{G_{2}+G_{2}^{\prime}}
$$

\section{Exemplary calculations}

Exemplary numerical calculations were carried out for the plate with the following geometrical dimensions: inner radius $r_{i}=0.2 \mathrm{~m}$, outer radius $r_{o}=0.5 \mathrm{~m}$, facing thickness $h^{\prime}=0.001 \mathrm{~m}$, core 
thickness $h_{2}=0.005 \mathrm{~m}, 0.01 \mathrm{~m}$ or $0.02 \mathrm{~m}$. The parameters of steel facing are: Young's modulus $E=2.1 \cdot 10^{5} \mathrm{MPa}$, Poisson's ratio $\nu=0.3$, mass density $\mu=7.85 \cdot 10^{3} \mathrm{~kg} / \mathrm{m}^{3}$. Polyurethane foam is the core material. Two kinds of foam presented in works by Romanów (1995) and Majewski and Mackowski (1975) have been accepted:

- with the values of constants $G_{2}, G_{2}^{\prime}, \eta^{\prime}, \mu_{2}$ presented in work by Romanów (1995) equal to: $G_{2}=15.82 \mathrm{MPa}, G_{2}^{\prime}=69.59 \mathrm{MPa}, \eta^{\prime}=7.93 \cdot 10^{4} \mathrm{MPa} \cdot \mathrm{s}, \mu_{2}=93.6 \mathrm{~kg} / \mathrm{m}^{3}$,

- with the data presented in work by Majewski and Mackowski (1975) the Kirchhoff's modulus equal to: $G_{2}=5 \mathrm{MPa}$, the creep function $\varphi=0.845\left(2-\mathrm{e}^{-0.36 t}-\mathrm{e}^{-0.036 t}\right)$ and mass density equal to: $\mu_{2}=64 \mathrm{~kg} / \mathrm{m}^{3}$.

The creep function presented in the work by Majewski and Mackowski (1975) allows for calculation of the values of elastic and viscous constants of the five-parameters rheological model composed of two Kelvin-Voigt models and the spring element connected in series. Because the solution presented in this paper is for the core material described by the three-parameters standard model (see, Fig. 2), hence the presented characteristic of function $\varphi(t)$ has been approximated by the function of the standard model. The numerical analysis has been carried out for the following values of standard constants of the plate core: $G_{2}=5 \mathrm{MPa}, G_{2}^{\prime}=3.13 \mathrm{MPa}$, $\eta^{\prime}=212.92 \cdot 10^{4} \mathrm{MPa} \cdot \mathrm{s}$, (Pawlus, 2010). According to the standard specification PN-84/B-03230 the value of Poisson's ratio is equal $\nu=0.3$. The values of Young's modulus calculated treating the foam material as an isotropic are equal, respectively, to $E_{2}=41.13 \mathrm{MPa}, E_{2}=13 \mathrm{MPa}$. The values of material constants $q_{1}^{p}, \tau_{1}^{G}$, expressed by Equations (5.2), in Prony series (see, Eq. (5.1)) are as follows:

- $q_{1}^{p}=0.615, \tau_{1}^{G}=26.19 \cdot 10^{4} \mathrm{~s}$ for the foam with $G_{2}=5 \mathrm{MPa}$,

- $q_{1}^{p}=0.185, \tau_{1}^{G}=928.46 \mathrm{~s}$ for the foam with $G_{2}=15.82 \mathrm{MPa}$.

Rapidly increasing loading acting on the edge is expressed by Equation (2.1). The rate of plate loading growth $s$ is equal for each numerically analysed plate. The value of the rate $s$ is the result of the following equation: $s=K_{7} p_{c r}$ (see Eq. (4.1)). The value of parameter $K_{7}$ is accepted as $K_{7}=20 \mathrm{~s}^{-1}$. Solving the eigenproblem, the value of critical stress $p_{c r}$ is $p_{c r}=217.32 \mathrm{MPa}$ calculated for the plate compressed on the inner perimeter with the facing thickness $h^{\prime}=0.001 \mathrm{~m}$, core thickness $h_{2}=0.01 \mathrm{~m}$ and value of core Kirchhoff's modulus $G_{2}=15.82 \mathrm{MPa}$ and $p_{c r}=$ $46.58 \mathrm{MPa}$ for the plate model with $N=26$ number of discrete points radially compressed on the outer perimeter with the number of buckling waves $m=7$, the facing thickness $h^{\prime}=0.001 \mathrm{~m}$, core thickness $h_{2}=0.005 \mathrm{~m}$ and value $G_{2}=15.82 \mathrm{MPa}$ (see Table 1 ).

The calculations by the finite difference method have been preceded by selection of the number $N$ of discrete points from numbers $N$, equal to $N=11,14,17,21,26$. The values of critical dynamic loads $p_{c r d y n}$ of plates with the viscoelastic core and values of critical static loads $p_{c r}$ have been evaluated. The numerical calculations show that the number $N=14$ allows us to achieve the accuracy up to $5 \%$ of technical error. The calculations have been carried out for this number. Table 1 presents exemplary results of the analysis.

The treating of the polyurethane foam as isotropic is one of the accepted assumptions in the description of the plate core material. In FEM numerical analysis, the value of the material Young's modulus has been calculated using the following equation: $G_{2}=E_{2} /[2(1+\nu)]$. Table 2 presents exemplary results of the critical time $t_{c r}$ and the critical additional deflection $w_{d c r}$ for the basic elastic plate model compressed on the inner edge with the core parameters: thickness $h_{2}=0.005 \mathrm{~m}$, Young's modulus $E_{2}$ presented in Table 2, Poisson's ratio $\nu=0.3$, Kirchhoff's modulus $G_{2}=15.82 \mathrm{MPa}$. The axisymmetric form $m=0$ of plate buckling has been examined.

In the wide range of the assumed values of Young's modulus $E_{2}$, the results for the critical time $t_{c r}$, and after calculations, also the values of the critical dynamic loads $p_{c r d y n}$ do not change. 
Table 1. Critical loads $p_{c r}, p_{c r d y n}$ of the plates with the viscoelastic core loaded on the outer edge $G_{2}=15.82 \mathrm{MPa} G_{2}^{\prime}=69.59 \mathrm{MPa}, \eta^{\prime}=7.93 \cdot 10^{4} \mathrm{MPa} \cdot \mathrm{s}, h_{2}=0.005 \mathrm{~m}, h^{\prime}=0.001 \mathrm{~m}$, $\mu_{2}=93.6 \mathrm{~kg} / \mathrm{m}^{3}, K_{7}=20 \mathrm{~s}^{-1}$

\begin{tabular}{|c|c|c|c|c|c|c|c|c|c|c|}
\hline \multirow{3}{*}{$m$} & \multicolumn{9}{|c|}{$N$} & \multicolumn{2}{|c|}{17} & \multicolumn{2}{|c|}{21} & \multicolumn{2}{|c|}{26} \\
\cline { 2 - 12 } & \multicolumn{2}{|c|}{11} & \multicolumn{2}{|c|}{14} & \multicolumn{2}{|c|}{17} \\
\cline { 2 - 11 } & $\begin{array}{c}p_{\text {cr }} \\
{[\mathrm{MPa}]}\end{array}$ & $\begin{array}{c}p_{\text {crdyn }} \\
{[\mathrm{MPa}]}\end{array}$ & $\begin{array}{c}p_{\text {cr }} \\
{[\mathrm{MPa}]}\end{array}$ & $\begin{array}{c}p_{\text {crdyn }} \\
{[\mathrm{MPa}]}\end{array}$ & $\begin{array}{c}p_{\text {cr }} \\
{[\mathrm{MPa}]}\end{array}$ & $\begin{array}{c}p_{\text {crdyn }} \\
{[\mathrm{MPa}]}\end{array}$ & $\begin{array}{c}p_{\text {cr }} \\
{[\mathrm{MPa}]}\end{array}$ & $\begin{array}{c}p_{\text {crdyn }} \\
{[\mathrm{MPa}]}\end{array}$ & $\begin{array}{c}p_{\text {cr }} \\
{[\mathrm{MPa}]}\end{array}$ & $\begin{array}{c}p_{\text {crdyn }} \\
{[\mathrm{MPa}]}\end{array}$ \\
\hline \hline 0 & 73.97 & 81.47 & 74.46 & 81.38 & 76.23 & 81.47 & 76.27 & 81.47 & 76.30 & 81.47 \\
\hline 1 & 70.28 & 79.42 & 70.73 & 79.42 & 72.51 & 79.51 & 72.56 & 79.51 & 72.59 & 79.51 \\
\hline 2 & 61.14 & 69.54 & 61.67 & 69.64 & 63.44 & 69.73 & 63.53 & 69.82 & 63.59 & 69.92 \\
\hline 3 & 53.01 & 67.96 & 53.39 & 68.05 & 55.10 & 68.15 & 55.21 & 68.33 & 55.30 & 68.33 \\
\hline 4 & 48.33 & 66.28 & 48.64 & 66.38 & 50.02 & 66.47 & 50.13 & 66.47 & 50.21 & 66.66 \\
\hline 5 & 46.11 & 53.43 & 46.36 & 52.59 & 47.45 & 52.50 & 47.55 & 52.21 & 47.62 & 52.03 \\
\hline 6 & 45.39 & 51.01 & 45.58 & 49.05 & 46.45 & 50.54 & 46.53 & 50.26 & 46.60 & 50.17 \\
\hline 7 & 45.60 & 47.84 & 45.75 & 47.47 & 46.45 & 47.65 & 46.52 & 47.93 & 46.58 & 47.84 \\
\hline 8 & 46.44 & 45.69 & 46.56 & 45.32 & 47.14 & 45.04 & 47.20 & 44.86 & 47.25 & 44.67 \\
\hline
\end{tabular}

Table 2. Values of the critical time $t_{c r}$ and maximum deflection $w_{d c r}$ depending on core Young's modulus $E_{2}$

\begin{tabular}{|l|c|c|c|c|c|}
\hline$E_{2}[\mathrm{MPa}]$ & 1.0 & 5.0 & 13.0 & 50.0 & 100.0 \\
\hline$t_{c r}[\mathrm{~s}]$ & 0.019 & 0.02 & 0.02 & 0.02 & 0.02 \\
\hline$w_{d c r} \cdot 10^{3}[\mathrm{~m}]$ & 4.14 & 4.48 & 4.15 & 3.85 & 3.73 \\
\hline
\end{tabular}

The values of the plate maximum critical deflection $w_{d c r}$ change according to prediction. The analysis shows that the accepted core material could be treated as an isotropic in the presented evaluation focused on the critical plate loads.

The calculation results of the plates with the standard core material subjected to momentary loads (duration in the range of $0.1 \mathrm{~s}$ ) do not differ significantly from the results for the plates with the elastic core. The time histories of deflections of the plates with the viscoelastic and elastic core for the number $m=0$ are presented in Fig. 4. The results are presented using two kinds of

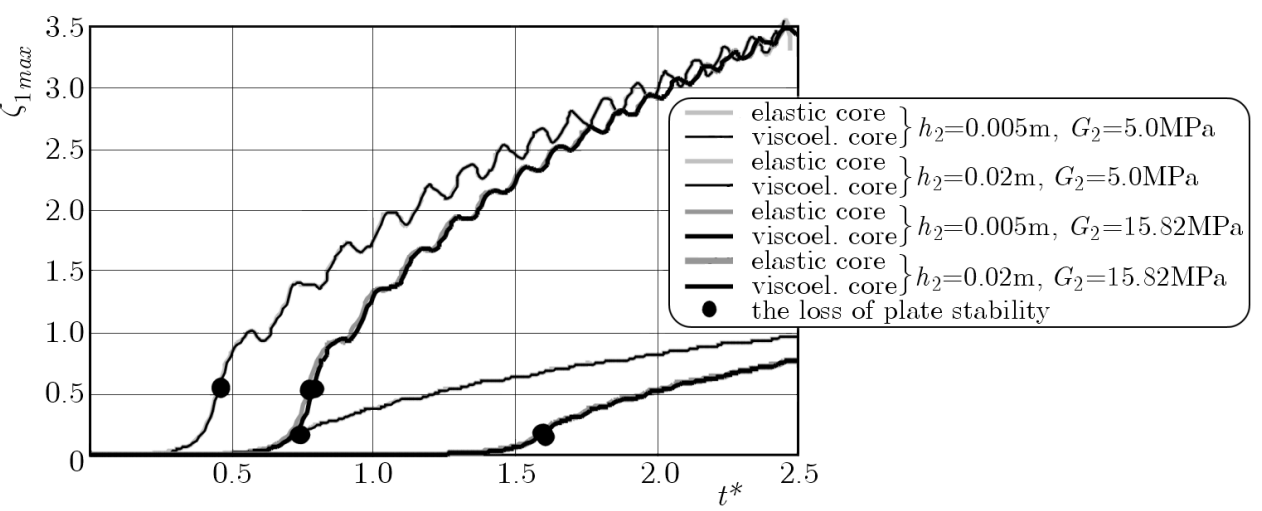

Fig. 4. A comparison of deflection time histories of plates loaded on the inner edge with the viscoelastic and elastic core

lines grey and black for the pairs: elastic and viscoelastic plate core with the same parameters $h_{2}, G_{2}$. The compatibility of deflection time histories is observed for the plates loaded on the inner perimeter with the viscoelastic and elastic core and compressed on the outer edge, too (see Fig. 5). The values of the critical time $t_{c r}$ and critical dynamic loads $p_{c r d y n}$ are practically the 
same. The critical deflections are comparable for the plates with core thickness: $h_{2}=0.005 \mathrm{~m}$ and $h_{2}=0.02 \mathrm{~m}$. Of course, the values of the critical time and load increase for the plate with the stiffer core (higher value of modulus $G_{2}$ and thickness $h_{2}$ ). Then, supercritical vibrations disappear.

Figure 5 shows characteristic time histories of plate deflections for a selected number of buckling $m=0, m=3, m=5, m=8$ in two respective groups. Some differences are observed in supercritical responses of the plates. The minimal value of plate critical parameters is for the waved plate with the number $m=5$ of buckling modes.
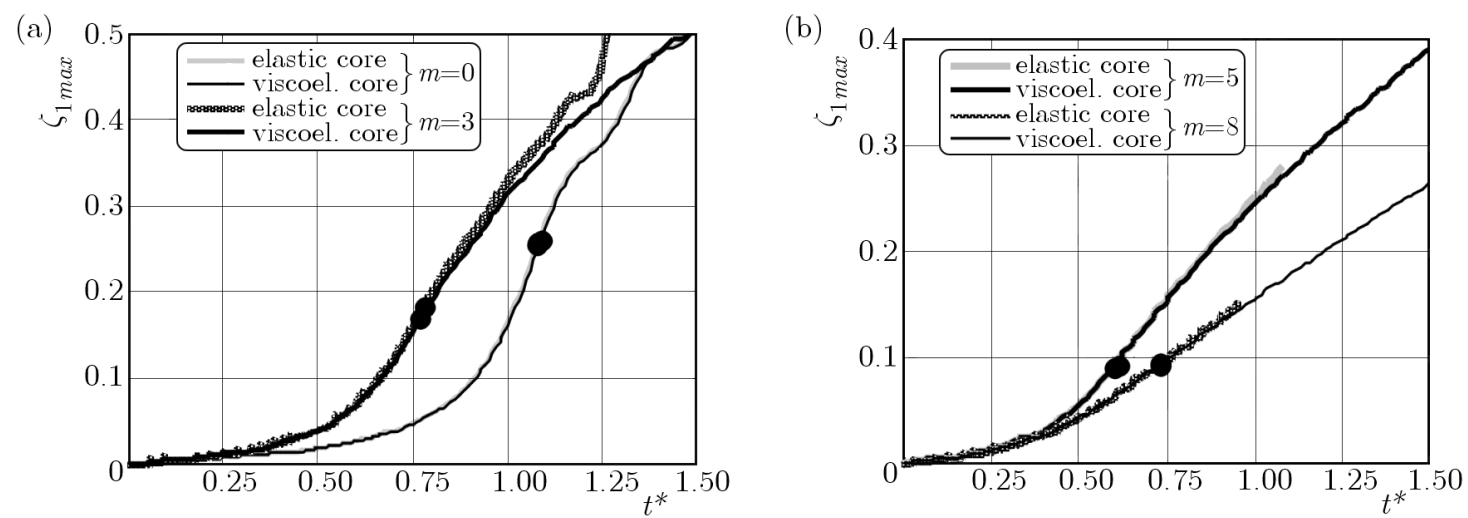

Fig. 5. A comparison of deflection time histories of plates $\left(G_{2}=5 \mathrm{MPa}, h_{2}=0.01 \mathrm{~m}\right)$ loaded on the outer edge with the viscoelastic and elastic core for the buckling wave number: (a) $m<4$, (b) $m>4$

The image of behaviour of the plates with the standard core is enriched by comparison with the results obtained for the plates with the core expressed by a simpler two-element model. The accepted in observations Maxwell model built of elastic and viscous elements connected in series takes attracts attention on the influence of the core material viscosity constant on the plate time histories of deflection. The numerical calculations have been carried out for quantities (3.5) assuming $G_{2}^{\prime}=0$. The comparison of deflection time histories of the plates compressed on the outer perimeter with the standard core $\left(G_{2}=15.82 \mathrm{MPa}\right)$ and the core expressed by the Maxwell model with parameters: $G_{2}=15.82 \mathrm{MPa}$ and viscosity constant $\eta^{\prime}=7.93 \cdot 10^{4} \mathrm{MPa} \cdot \mathrm{s}$ is shown in Fig. 6. The results are presented for selected buckling forms, which entirely show

(a)

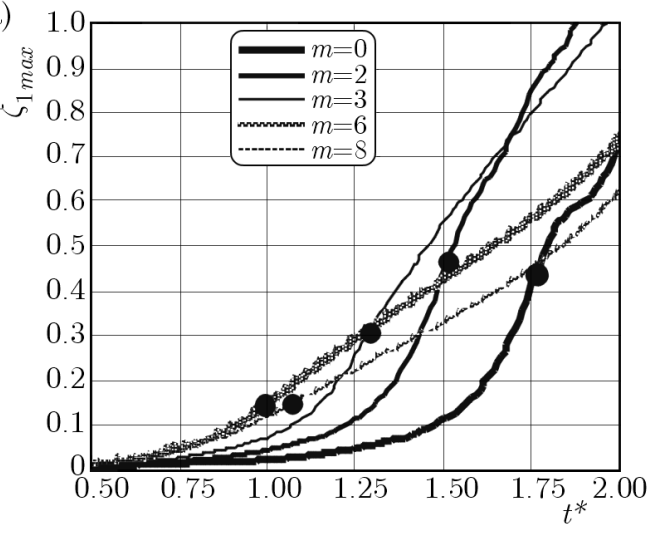

(b)

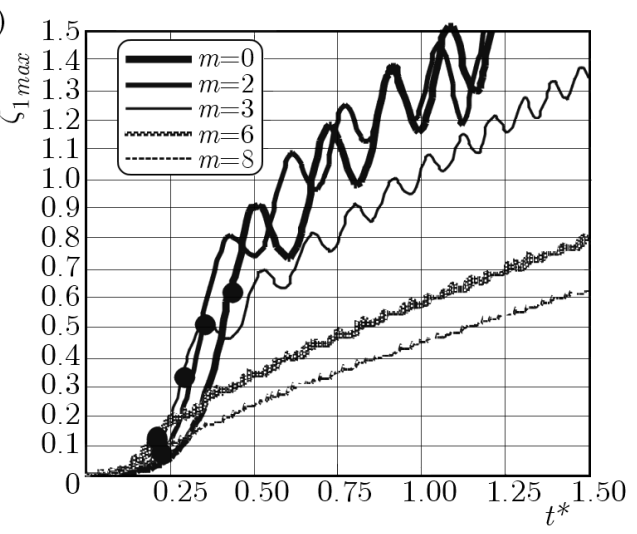

Fig. 6 . The time histories of deflections of plates $\left(G_{2}=15.82 \mathrm{MPa}, h_{2}=0.005 \mathrm{~m}\right)$ compressed on outer edge with: (a) standard core, (b) Maxwell core

the character of curves. The standard model of solid body clearly extends the time to the loss of plate stability. In the case of the Maxwell model, the character of time histories of deflections of the plates with various numbers of circumferential waves $m$ is comparable - increasing in 
the plate loading time. One can observe that the deflection time histories for the plate with the standard core model differ for plates with higher and lower numbers of the buckling mode. Figure 5 shows this character of curves too.

Figure 7 shows the comparison of curves $\zeta_{1 \max }=f\left(t^{*}\right)$ of the plates with the elastic and viscoelastic core compressed on the outer edge with the number of circumferential waves corresponding to the minimal values of the critical dynamic load for different values of material parameters and core thickness. The same lines (gray and black) are used to present the results for the plates with the elastic and viscoelstic core. The diagram presents the results for the plates with the Maxwell core, too. For this simple model, the change of material parameters $\left(G_{2}=15.82 \mathrm{MPa}, \eta^{\prime}=7.93 \cdot 10^{4} \mathrm{MPa} \cdot \mathrm{s}\right.$ and $\left.G_{2}=5 \mathrm{MPa}, \eta^{\prime}=212.92 \cdot 10^{4} \mathrm{MPa} \cdot \mathrm{s}\right)$ does not influence the deflection time histories and the critical time. Whereas, the plate core thickness has a great importance. The results show the relevant sensibility of the plate structure with three elements and the standard core on the material and geometrical parameters. It confirms an obvious and better approximation of the structure behaviour of the expected responses of the actual object. The results obtained for the plate compressed on the outer edge confirm earlier observations that the critical time prolongs for the plates with the stiffer core. For these plates, the minimal critical dynamic load is for the mode $m=5-7$. It depends on the core parameters $G_{2}, h_{2}$. The value of the critical additional deflection is about 0.1 of the total plate thickness (see Eq. (4.1)). It is slightly higher for the plate with the modulus $G_{2}=15.82 \mathrm{MPa}$.
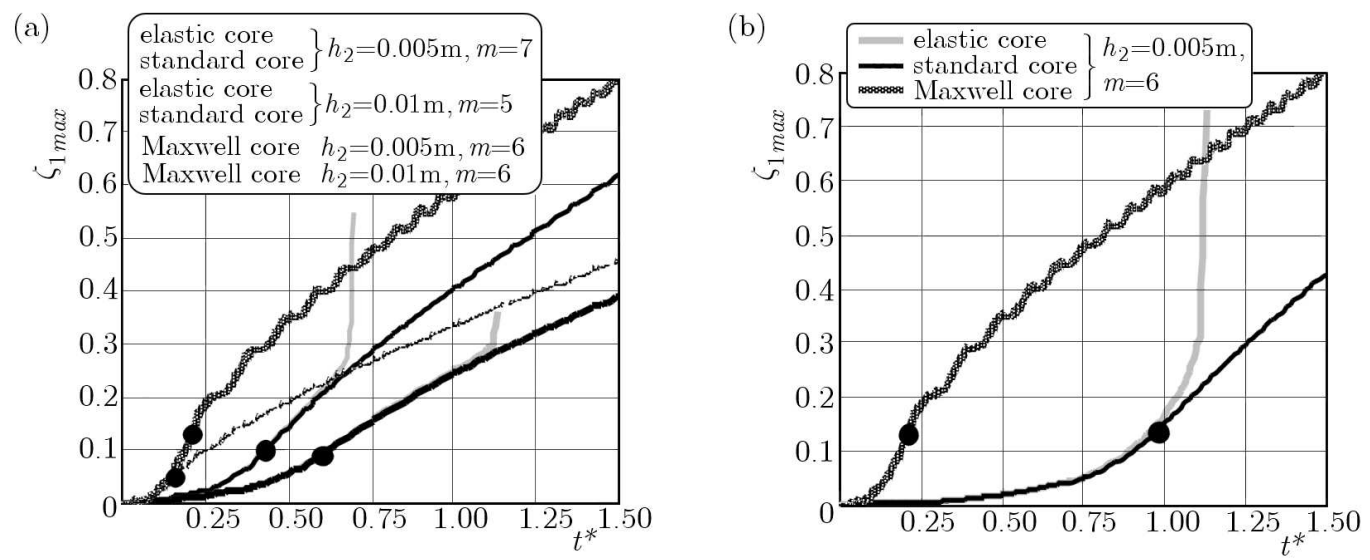

Fig. 7. Time histories of deflections of plates compressed on the outer edge for the elastic standard and Maxwell core with: (a) $G_{2}=5 \mathrm{MPa}$, (b) $G_{2}=15.82 \mathrm{MPa}$

The viscoelastic properties of the foam material relevantly depend on the viscosity constant $\eta^{\prime}$. The deflection results for the plates with the viscoelastic core of significantly lower viscosity constant $\eta^{\prime}$ differ. Figure 8 shows the deflection time histories of the plates loaded on the inner edge with various values of the viscosity constant $\eta^{\prime}$ of the viscoelastic core material.

Large differences could be observed for the core material with very small values of the constant $\eta^{\prime}$ within the range $7.93 \cdot 10^{-2}\left(10^{-3}\right) \mathrm{MPa} \cdot \mathrm{s}$. The time to the loss of plate stability shortens. The increase in values of the constant $\eta^{\prime}$ does not change the critical or supercritical deflections of the plates with the viscoelastic core. They are compatible with the results obtained for the plates with the elastic core.

The numerical FEM calculations of the simplified plate model confirm these observations. The critical dynamic loads distribution depending on values of the constant $\eta^{\prime}$ is presented for two kinds of the core viscoelastic materials in Fig. 9. The plates are loaded on the inner edge. A large theoretical decrease in values of the number $\eta^{\prime}$ up to a value about $10^{-8}$ times lower influences the contraction of the time to the loss of dynamic stability and decreases critical dynamic loads. These observations have an important practical meaning. In a wide range of accepted values of 


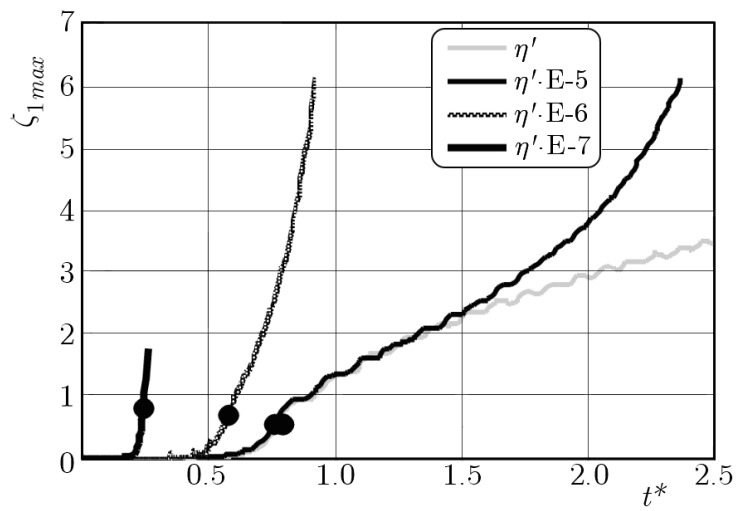

Fig. 8. The influence of viscosity constant $\eta^{\prime}$ on the deflection time histories of plate $\left(G_{2}=15.82 \mathrm{MPa}\right.$, $h_{2}=0.005 \mathrm{~m}$ ) loaded on the inner edge

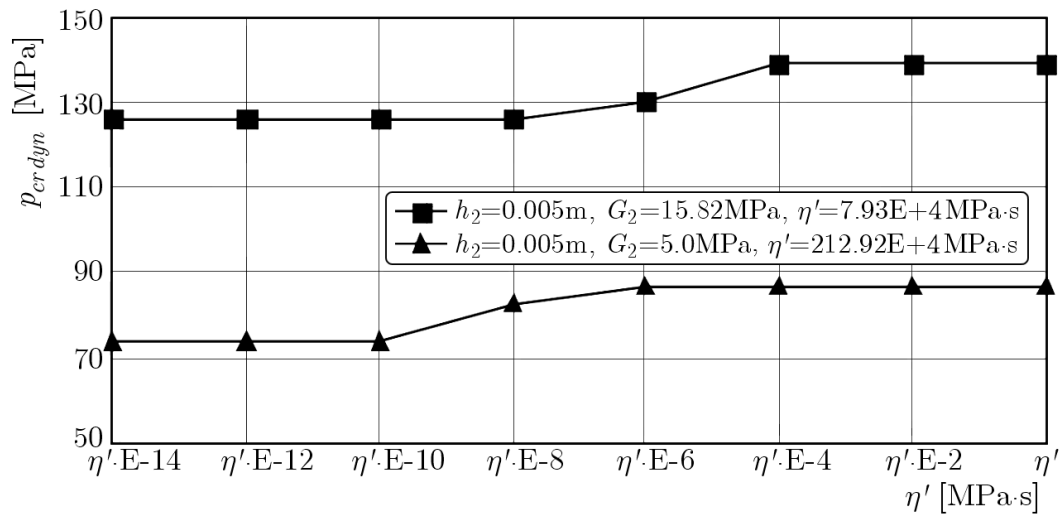

Fig. 9. Influence of the viscosity constant $\eta^{\prime}$ on the critical dynamic loads $p_{\text {crdyn }}$ of the FEM plate model loaded on the inner edge

the viscosity constant, the dynamic response of the plate subjected to quickly increasing loads does not change. Here, it should be noticed that for true materials, precise determination of viscous material parameters is a great problem. Smaller sensitiblity to fluctuation of values of the viscoelastic material parameters seems to be the advantageous dynamical behaviour of such plates.

Exemplary time histories of deflections and velocity of deflections as well as critical deformations of the FEM simplified plate model with the elastic and viscoelastic core with $\eta^{\prime}=212.92 \cdot 10^{-6}\left(10^{-10}\right.$ times decrease $)$ are presented in Fig. 10. In general, there are no differences in the character of curves. A slightly less critical time and also dynamic load with higher deflection are noticed for the plate with a strongly reduced value of the viscosity constant $\eta^{\prime}$. For a higher parameter $\eta^{\prime}$, the response of the plate with the viscoelastic core is consistent with that with the elastic one.

The influence of rheological properties of the core material on the increase of plate deflections is observed for the plates subjected to the load constant in time. The supercritical response has been analysed for both plates with the viscoelastic and elastic core compressed on the inner or outer edge in the case of the load constant in time. The assumed load function is expressed as follows

$$
p(t)= \begin{cases}s t & \text { for } t \leqslant n t_{c r} \\ n p_{\text {crdyn }} & \text { for } t>n t_{c r}\end{cases}
$$

where $t_{c r}$ is the critical time of the loss of plate dynamic stability, $n$ - assumed number. 
(a)
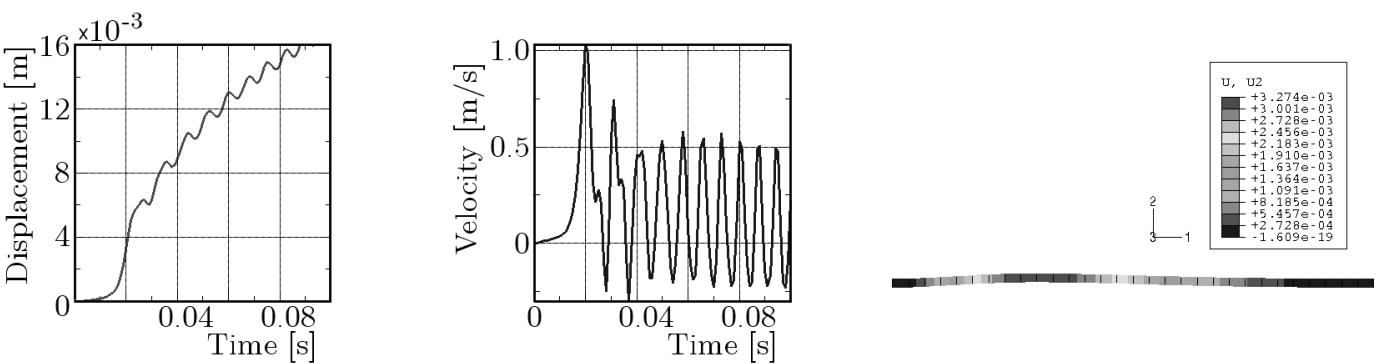

(b)
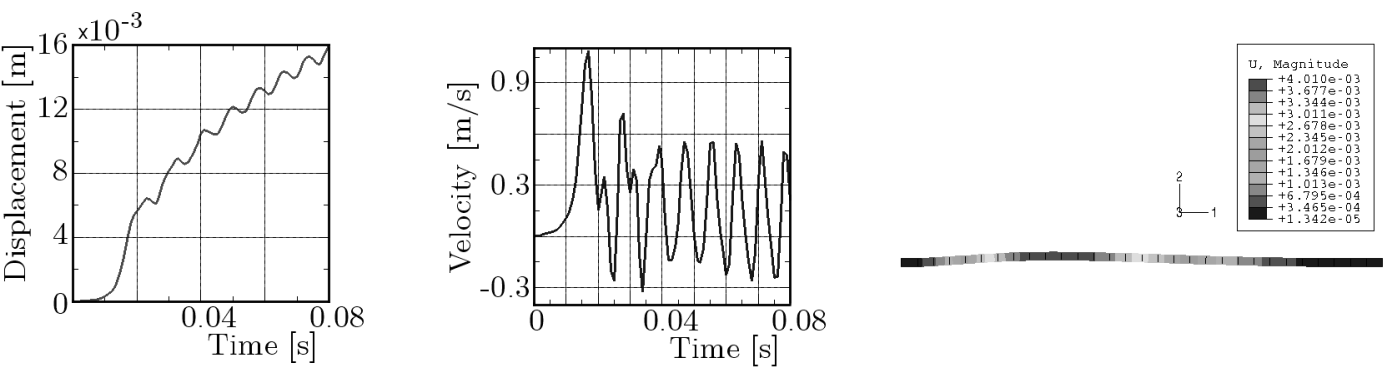

Fig. 10. Time histories of deflections, velocity of deflections and buckling forms of the plates $\left(G_{2}=5 \mathrm{MPa}, h_{2}=0.005 \mathrm{~m}\right)$ with: (a) elastic core, (b) viscoelastic core loaded on the inner edge

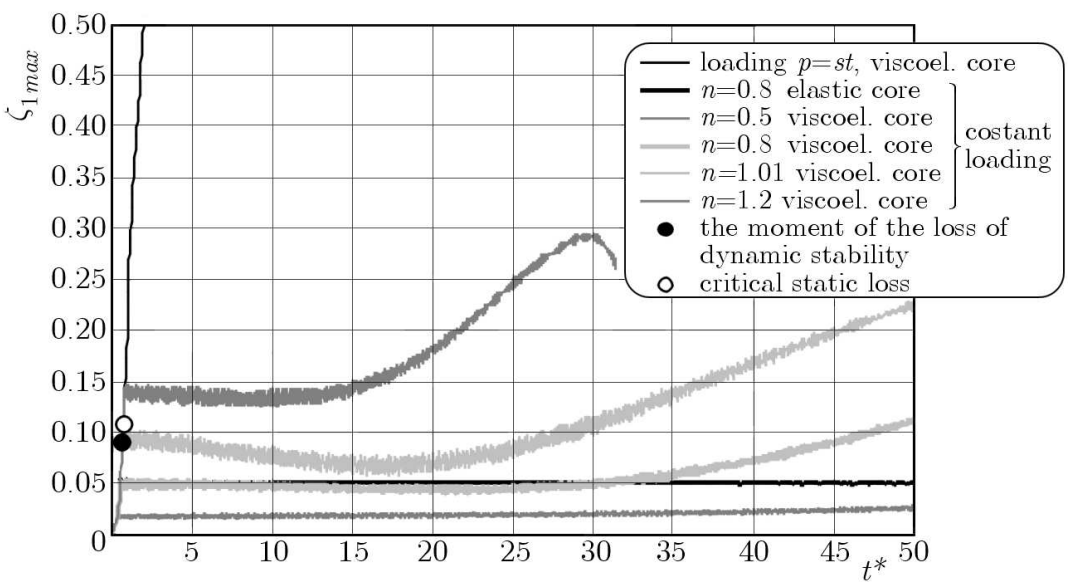

Fig. 11. Time histories of deflections of plates $G_{2}=5 \mathrm{MPa}, h_{2}=0.01 \mathrm{~m}, m=5$ with the elastic and viscoelastic core subjected to the load constant in time acting on the outer edge

Figure 11 shows the time histories of deflections of the plates with the elastic and viscoelastic core compressed on the outer edge with the waved form of predeflection and buckling. The number $m$ is equal 5 . The value of the number $n$ is equal $0.5,0.8$ (undercritical load) and $n=1.01,1.2$. Additionally, Fig. 11 presents the curve $\zeta_{1 \max }=f\left(t^{*}\right)$ obtained for a linear $(p=s t)$ increase in the load acting on the plate with the viscoelastic core. The marked points show the moment of the loss of plate dynamic stability and static stability after suitable miscalculations in the value of the critical static load. The course of deflection time histories of the plates with the elastic and viscoelastic core under increasing linear load are consistent. The differences could be observed in the range for the loading constant in time. In a short time $(t=2.5 \mathrm{~s})$ of the loading, an increase in the deflection of the plate with the viscoelastic core can be significant. An increase in the deflection rises in the critical $(n=0.8,1.01)$ and supercritical $(n=1.2)$ range of plate operation. The sensitivity of the plate structure with the viscoelastic core material subjected to the load constant in time is clear. It is interesting that the course of these curves is observed for the plates examined in the short loading time. Doubtlessly, a quick increase in the dynamic loads, which involves the basic part of plate operation, influences such behaviour. One can find 
that the course of curves for the plates subjected to the load constant in time corresponds to the characteristic rheological curve with zones of the stationary and final creep.

The influence of dynamics of the increasing loading on the course of constant deflections of the plate with the viscoelastic core compressed on the outer perimeter is shown in Fig. 12. The rate of loading growth expressed by the parameter $K_{7}$ is not out of meaning in the response of the examined plate under the supercritical load (the number $n$ is equal 1.01) constant in time. The dynamic behaviour with an increase in deflections of the plate initially linearly loaded with different values of the parameter $K_{7}$ in a longer time of the loading with a constant value is observed. The thickness $h_{2}$ of the plate with the viscoelastic core has here the influence, too. The results confirm dynamic sensitivity of the sandwich plate with the viscoelastic core. They also show importance of the general solution with the accepted description of the physical relations for the core rheological material in the analysed problem.

(a)

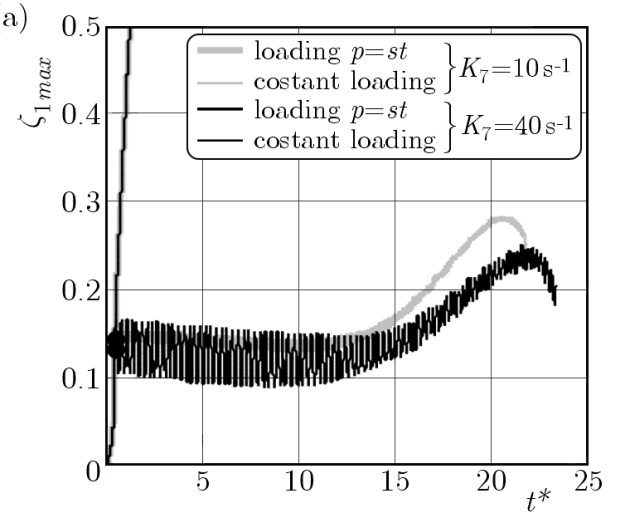

(b)

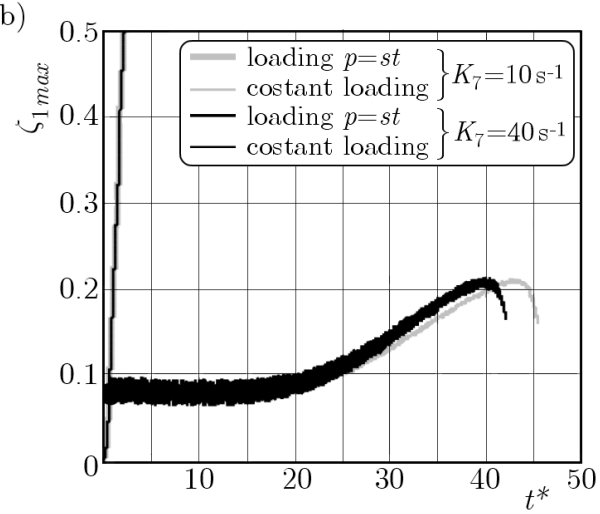

Fig. 12. Time histories of deflections of plates with the viscoelastic core subjected to the load constant in time acting on the outer edge depending on the rate of loading growth $K_{7}$ for different core thicknesses $h_{2}$ : (a) $h_{2}=0.005 \mathrm{~m}, G_{2}=5 \mathrm{MPa}, n=1.01, m=7$, (b) $h_{2}=0.01 \mathrm{~m}, G_{2}=5 \mathrm{MPa}$, $n=1.01, m=6$

\section{Conclusion}

Summarizing the presented results and observations, it could be stated that, in general, the calculations of plates with an viscoelastic core could be replaced by calculations of similar plates but with an elastic core. Then, the solution of the problem could be significantly easier. However, the presented results show plate responses to its rheological properties. It has been observed that the plate behaviour differ for plates subjected to short critical time loadings and for plates loaded longer. The reactions on viscoelastic material parameters of the plate structure under longer duration of loading are quite clear. The indicate that the use of viscoelastic expressions in the plate physical relations is relevant. Then, the practical calculations become more difficult and should be carried out solving a more complicated problem formulation. Additional plate parameters connected with the effects of external factors like higher temperature make the problem more complex, which requires the achievement of the generalized solution.

The computational results also showed the influence of dynamics of the preliminary loading on the growth of rheological deflections of plates loaded constant in time. Both loading time histories expressed by the assumed function, in this paper by Eq. (6.1), as well as the speed of loading growth have an important meaning in this problem. A detailed analysis could expand the knowledge about dynamic behaviour of such a plate structure.

The carried out numerical experiments allow one to formulate the following statements:

- the use of proper constitutive relations for the plate material with rheological properties is essential in the solution to the dynamic deflection problem, 
- determination of the material model of the viscoelastic layer and the values of its constants is a problem in itself. This multiparameter problem requires some compromise between the attempt to find an exact problem description and the assumption of a rationally sophisticated one,

- action of loads in longer duration on the plate structure identifies its rheological properties.

\section{References}

1. Chen Y.R., Chen L.W., Wang C.C., 2006, Axisymmetric dynamic instability of rotating polar orthotropic sandwich annular plates with a constrained damping layer, Composite Structures, 73, $2,290-302$

2. Dumir P.C., Shingal L., 1985, Axisymmetric postbuckling of orthotropic thick annular plates, Acta Mechanica, 56, 229-242

3. Hibbitt, Karlsson \& Sorensen, Inc., 2000, ABAQUS/Standard User's Manual, version 6.1

4. Krizhevsky G., Stavsky Y., 1996, Refined theory for vibrations and buckling of laminated isotropic annular plates, International Journal of Mechanical Sciences, 38, 5, 539-555

5. Majewski S., MaćKowski R., 1975, Creep of foamed plastics used as the core of a sandwich plate (in Polish), Inżynieria i Budownictwo, 3, 127-131

6. PAwlus D., 2010, Dynamic stability of three-layered annular plates with a viscoelastic core (in Polish), Scientific Bulletin of the Technical University of Eódź, 1075

7. PAwlus D., 2011a, Critical loads calculations of annular three-layered plates with soft elastic or viscoelastic core, Archives of Civil and Mechanical Engineering, XI, 4, 993-1009

8. PAwlus D., 2011b, Dynamic stability of three-layered annular plates with wavy forms of buckling, Acta Mechanica, 216, 123-138

9. PAwlus D., 2011c, Solution to the problem of axisymmetric and asymmetric dynamic instability of three-layered annular plates, Thin-Walled Structures, 49, 660-668

10. Paydar N., 1990, Axisymmetric buckling of an annular sandwich plate of varying thickness, Composite Structures, 15, 149-159

11. Romanów F., 1995, Strength of Sandwich Constructions (in Polish), WSI in Zielona Góra, Poland

12. SkrzypeK J., 1986, Plasticity and Creep (in Polish), Warszawa, PWN

13. Standard, PN-84/B-03230, Lightweight curtain walls and roofs of sandwich and rib panels. Static calculation and design (in Polish), Standardization Publishers "Alfa", Warszawa, 1985

14. Tanov R., Tabiei A., 1998, Static and dynamic buckling of laminated composite shells, Proceedings of the 5-th International LS-DYNA Users Conference, South Field, MI.

15. Volmir C., 1967, Stability of Deformed Systems (in Russian), Moskwa, Science

16. Volmir C., 1972, Nonlinear Dynamics of Plates and Shells (in Russian), Moskwa, Science

17. WAng H.J., Chen L.W., 2002, Vibration and damping analysis of a three-layered composite annular plate with a viscoelastic mid-layer, Composite Structures, 58, 563-570

18. WAng H.J., Chen L.W., 2004, Axisymmetric dynamic stability of rotating sandwich circular plates, Journal of Vibration and Acoustics, 126, 407-415 